\title{
Magnetic Resonance Arthrography in Rugby Players Undergoing Shoulder Stabilization for Glenohumeral Instability: Professionals Have Higher Frequencies of More Pathologies
}

\author{
Martin S. Davey, M.B., B.Ch., M.Ch., Eoghan T. Hurley, M.B., B.Ch., M.Ch., \\ Daire J. Hurley, M.B., B.Ch., Leo Pauzenberger, M.D., and Hannan Mullett, M.Ch., F.R.C.S.
}

Purpose: To quantify the magnetic resonance arthrography (MRA) findings in rugby players during preoperative workup for anterior surgical stabilization for glenohumeral instability. Methods: All patients who underwent glenohumeral instability surgery in our institution between 2008 and 2018 were considered for inclusion. Rugby players were identified using the patient's medical notes, with subsequent identification of all professional players. All rugby player's preoperative MRA findings were recorded and analyzed. Results: Overall, 267 rugby players were included, 261 of whom were male $(97.8 \%)$, with a mean age of 22.7 years (range $13-55$ years). There were 58 professional rugby players $(21.7 \%)$. The mean number of pathologies in nonprofessional rugby players was 5.0 pathologies versus 6.2 pathologies in the professional rugby players, with a significant difference in nonprofessional rugby players with up to 3 pathologies versus professional rugby players $(26.3 \%$ vs $10.3 \%, P=.01)$. Professional rugby players had a statistically significant increased incidence of bicipital tendon lesions $(25.9 \%$ vs $13.9 \%, P=.009)$, acromioclavicular joint degeneration $(60.3 \%$ vs $42.1 \%, P=.016)$, glenohumeral bone loss $(87.9 \%$ vs $69.9 \%, P=.006)$, and degenerative changes $(67.2 \%$ vs $44.0 \%, P=.002)$ on their MRAs. Conclusions: Rugby players undergoing surgical stabilization for glenohumeral instability often have a significant number of pathologies identified on MRA at the time of surgery. Professional rugby players showed concerningly greater frequencies of early degenerative changes when compared with nonprofessional rugby players. Level of Evidence: III; Retrospective Cohort Study

G lenohumeral instability is a common clinical problem among collision athletes, with an incidence being reported as 14,800 per 100,000 adolescent rugby players, compared with 8 per 100,000 in the general population. ${ }^{1-5}$ This injury has become increasingly more common in rugby players over the past 2 decades, with the literature reporting high incidences of

From the Sports Surgery Clinic (M.S.D., E.T.H., D.J.H., L.P., H.M.); and Royal College of Surgeons in Ireland (M.S.D., E.T.H.), Dublin, Ireland.

The authors report that they have no conflicts of interest in the authorship and publication of this article. Full ICMJE author disclosure forms are available for this article online, as supplementary material.

Received June 2, 2020; accepted December 20, 2020.

Address correspondence to Eoghan T. Hurley, M.B., B.Ch., M.Ch., Sports Surgery Clinic, Santry, Dublin 9, Ireland. E-mail: eoghanhurley@rcsi.ie

(C) 2021 THE AUTHORS. Published by Elsevier Inc. on behalf of the Arthroscopy Association of North America. This is an open access article under the CC BY-NC-ND license (http://creativecommons.org/licenses/by-nc-nd/4.0/). 2666-061X/20976

https://doi.org/10.1016/j.asmr.2020.12.006 glenohumeral instability in collision-sport athletes, with rates of 0.40 injuries per 1000 athlete exposures.

In modern practice, magnetic resonance (MR) has been used as a gold-standard preoperative diagnostic investigation in evaluating labral injuries. ${ }^{7}$ Magnetic resonance arthrography (MRA) with contrast has been found to be the most useful single modality for evaluating labral lesions. ${ }^{8}$ Although not as immediately useful as computed tomography for evaluating bone loss, MR has been found to be adequate for surgical decision-making and has advantages of being without high radiation while allowing excellent soft-tissue visualization. ${ }^{9}$

The frequency of pathologic MR findings in rugby players undergoing stabilization for glenohumeral instability is unknown. Therefore, the purpose of this study was to quantify the MRA findings in rugby players during preoperative workup for anterior surgical stabilization for glenohumeral instability. Our hypothesis was that rugby players would have a substantial number of pathologies identified on their 
MRAs, with professional rugby players having a significantly greater frequency of pathologic findings on their MRAs.

\section{Methods}

Using an electronic database, we retrospectively identified all patients who underwent arthroscopic Bankart repair, open Bankart repair, or a Latarjet procedure by a single surgeon between 2008 and 2018 . Inclusion criteria for this study were the following: rugby union athlete, presented with anterior shoulder instability, and underwent a preoperative MRA in our institution. Included patients were subsequently assigned a randomized study number to maintain anonymity throughout the study.

A 3-Tesla magnet was used for all MRAs (Twinspeed 8; GE Medical Systems, Milwaukee, WI). A dedicated shoulder surface coil was used, and patients were positioned with the shoulder in a neutral position. T1weighted fat-saturated coronal, sagittal, and axial images and $\mathrm{T} 2$-weighted fat-saturated coronal images were obtained. The MRAs were assessed at the time by a fellowship-trained musculoskeletal radiologist.

Pathologies were defined to include the following: (1) labral tear, (2) SLAP tear, (3) glenolabral articular disruption lesion, (4) humeral avulsion of the glenohumeral ligament, (5) anterior labral periosteal sleeve avulsion, (6) acromioclavicualar joint degenerative, (7) bicipital lesion, (8) rotator cuff lesion, (9) Hill-Sachs (HS) lesion, (10) reverse HS lesion, (11) degenerative changes, (12) Bankart lesion, and (13) bony cysts. Glenoid bone loss was defined to include (1) Bankart lesions and (2) acromioclavicular joint degeneration, whereas glenohumeral bone loss was defined to include (1) glenoid bone loss, (2) HS lesions, and (3) reverse HS lesions.

\section{Statistical Analysis}

Qualitative statistical analysis was performed using the SPSS (version 22.0. IBM Corp., Armonk, NY). $\chi^{2}$ analysis was performed comparing pathologies found between professional rugby players and nonprofessional rugby players.

\section{Results}

\section{Patient Demographics}

There were 267 rugby players who underwent surgical stabilization for glenohumeral instability enrolled in the study, 261 of whom were male $(97.8 \%)$. The mean age of the rugby players was 22.7 years (range 13-55 years). There were 58 professional rugby players $(21.7 \%)$ who underwent surgical stabilization for glenohumeral instability enrolled in the study, 56 of whom were male $(96.5 \%)$. The mean age of the professional rugby players was 22.0 years (range 1732). These findings are further illustrated in Table 1.

\section{MR Findings}

Overall, 249 rugby players had labral tears (93.3\%), including 87 SLAP lesions (32.6\%) identified on MRA. There were 109 rugby players with rotator cuff lesions $(40.8 \%), 44$ with bicipital tendon lesions $(16.5 \%)$, and 163 HS lesions $(61.0 \%)$. In addition, 132 rugby players had anterior glenoid bone loss $(49.4 \%), 197$ had glenohumeral bone loss $(73.8 \%)$, and 104 had bone loss in both anatomical areas $(39.0 \%)$ identified on MR. There were 131 with degenerative changes $(49.1 \%), 123$ with acromioclavicular joint degeneration $(46.1 \%)$, and 61 with bony cysts of the glenoid or humeral head $(22.8 \%)$ identified on MR. These findings are further illustrated in Table 2.

\section{Comparison Between Professional and Nonprofessional Rugby Players}

Overall, 58 professional rugby players were compared with the 209 nonprofessional rugby players. There was a nonstatistically significant difference between the mean number of pathologies described on their MRAs in the nonprofessional rugby players and the professional rugby players; 5.0 pathologies versus 6.2 pathologies, respectively $(P>.05)$. Nonprofessional rugby players were significantly more likely to have up to 3 pathologies described on their MRA (when compared with professional rugby players; $26.3 \%$ vs $10.3 \%, P=$ $.01)$. There were no other significant differences in the combined numbers of pathologies between the professional and nonprofessional rugby players identifiable on MRA ( $P>.05$ for all). Professional rugby players had a statistically significant increased prevalence of bicipital tendon lesions $(25.9 \%$ vs $13.9 \%, P=.009)$, acromioclavicular joint degeneration $(60.3 \%$ vs $42.1 \%$, $P=.01)$, degenerative changes $(67.2 \%$ vs $14.8 \%, P=$ $.002)$, and glenohumeral bone loss $(87.9 \%$ vs $69.9 \%$, $P=.006)$ identified on MRA. There were no other significant differences in the prevalence of other pathologies between the professional and nonprofessional rugby players $(P>.05$ for all). These findings are further illustrated in Fig 1.

Table 1. Patient Demographics

\begin{tabular}{llcc}
\hline \multicolumn{1}{c}{ Characteristic } & Number $(\%)$ & Nonprofessionals & Professionals \\
\hline Number & 267 & $209(78.3 \%)$ & $58(21.7 \%)$ \\
Male & $261(97.8 \%)$ & $205(98.1 \%)$ & $56(96.5 \%)$ \\
Mean age, y & 22.66 & 22.83 & 22.04 \\
Left shoulders & $140(52.4 \%)$ & $106(50.7 \%)$ & $34(58.6 \%)$ \\
Right shoulders & $127(47.6 \%)$ & $103(49.3 \%)$ & $24(41.4 \%)$ \\
Arthroscopic Bankart & $175(65.5 \%)$ & $131(62.7 \%)$ & $44(75.9 \%)$ \\
Open Bankart & $15(5.6 \%)$ & $13(6.2 \%)$ & $2(3.5 \%)$ \\
Latarjet & $77(28.8 \%)$ & $60(28.7 \%)$ & $17(29.3 \%)$ \\
\hline
\end{tabular}


Table 2. Comparison of Pathologies Found Between Professional and Nonprofessional Rugby Players

\begin{tabular}{|c|c|c|c|c|}
\hline Associated Lesions & Total & Professionals & Nonprofessionals & $P$ value \\
\hline Total & 267 & $58(21.7 \%)$ & $209(78.3 \%)$ & - \\
\hline Age & 22.66 & 22.03 & 22.89 & - \\
\hline Left & 140 & $34(58.6 \%)$ & $106(50.7 \%)$ & - \\
\hline Labral tear & 249 & $57(98.3 \%)$ & $191(91.4 \%)$ & .07 \\
\hline SLAP tear & 87 & $20(34.5 \%)$ & $67(32.1 \%)$ & .73 \\
\hline GLAD lesion & 30 & $6(10.3 \%)$ & $24(11.5 \%)$ & .81 \\
\hline AC joint degeneration & 123 & $35(60.3 \%)$ & $88(42.1 \%)$ & .014 \\
\hline Bicipital lesion & 44 & $15(25.9 \%)$ & $29(13.9 \%)$ & .009 \\
\hline Rotator cuff lesion & 109 & $29(50.0 \%)$ & $80(38.3 \%)$ & .11 \\
\hline HS lesion & 163 & $35(60.3 \%)$ & $128(61.2 \%)$ & .42 \\
\hline RHS lesion & 17 & $5(8.6 \%)$ & $12(5.7 \%)$ & .43 \\
\hline Degenerative changes & 131 & $39(67.2 \%)$ & $92(44.0 \%)$ & .002 \\
\hline
\end{tabular}

AC, acromioclavicular; ALPSA, anterior labral periosteal sleeve avulsion; GLAD, glenolabral articular disruption; HAGL, humeral avulsion of the glenohumeral ligament; HS, Hill-Sachs; RHS, reverse Hill-Sachs.

\section{Discussion}

The most important finding in this study was that rugby players undergoing surgical stabilization for glenohumeral instability had a high number of pathologies identified on their MRAs. Both the professional and nonprofessional rugby players had multiple pathologies identified on their MRAs, with labral tears seen in more than $90 \%$ of rugby players included. This study demonstrated that significantly greater frequencies of degenerative changes, glenohumeral bone loss, acromioclavicular degeneration, as well as bicipital tendon lesions were identified on the MRAs of the professional rugby players when compared with those of the nonprofessional rugby players.

The incidence of glenohumeral instability is significantly greater in rugby players, with studies describing rates of glenohumeral instability as more than 1500 times more likely to occur in rugby players when compared with the general population. ${ }^{1,10-12}$ The reason for this is thought to be primarily due to the increased number of collisions, as well as a number of other factors. Second, the establishment of the professional playing era in the mid-1990s has seen a significant increase in mean elite rugby players' mass, from 88 kilograms in the amateur era to 104 kilograms today. ${ }^{13}$ Third, rugby laws have been constantly developed over this period, with the ball now being in play for almost twice as long with 3 times as many tackles per games when compared with the nonprofessional era. ${ }^{14,15}$ The authors of this study believe that a combination of the aforementioned factors have resulted in the greater frequencies of shoulder pathologies being reported on MRA in not only the professional rugby player, but the nonprofessional or previously deemed "social" player,
Fig 1. Comparison of the percentage of combined pathology frequencies identified on magnetic resonance arthrography for the professional and nonprofessional rugby players.

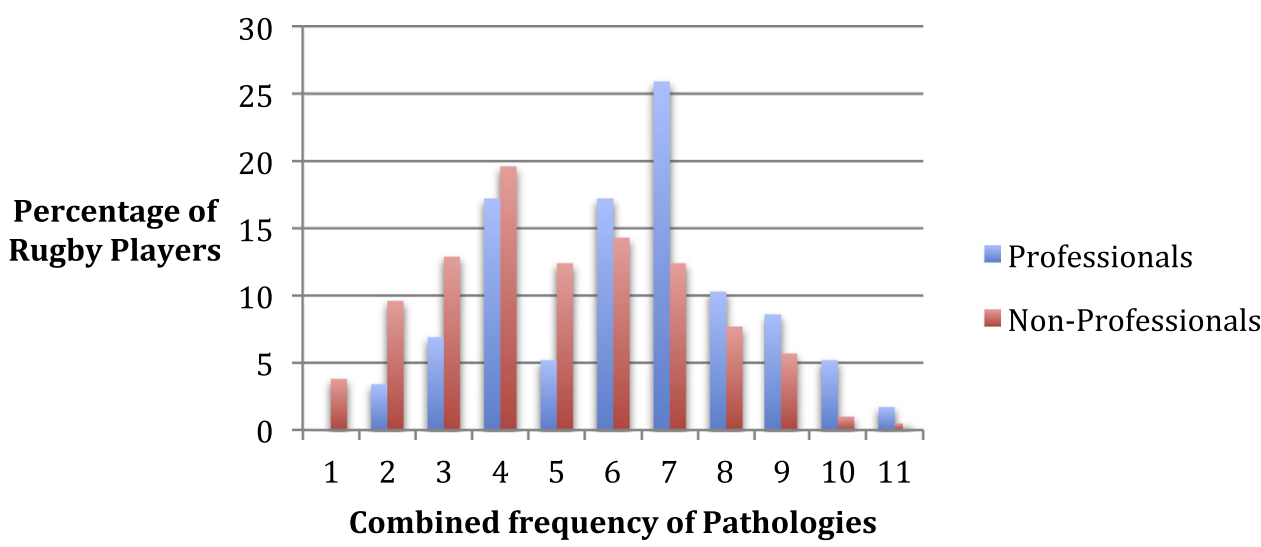


raising concern for these young athletes across the board.

Rugby has seen a continuous evolution of the tackle and "poacher" mechanisms of defensive play, which has subsequently led to an increase in frequency of defending players using their shoulders for head-on collisions at greater forces. ${ }^{16-18}$ These mechanisms are also the most likely etiology in developing a single episode of glenohumeral dislocation, as well as recurrent dislocations and instability. The mechanism of this pathology in cases of glenohumeral instability involve the humeral head being displaced anteriorly from the glenoid labrum; this theoretically reinforces the rationale for the high frequencies of bony and chondral pathologies in collision athletes such as rugby players. Although the literature widely reports that American football athletes have a similarly increased risk of glenoid labral tears (among other pathologies) secondary to collisions, ${ }^{19-21}$ Knapik et al. ${ }^{22}$ reported that not only unilateral tears are commonplace in this cohort but so too are recurrent and bilateral tears on MR imaging. Despite this, they found that this did not directly affect future short-term participation in the National Football League. $^{22}$

Following shoulder dislocations, rugby players have an average time of 12 weeks before they are able to return to play. ${ }^{3}$ Such injuries affect professional rugby players in a similar manner, with a mean of $18 \%$ of a professional rugby club's registered rugby players having a shoulder injury at any one time. ${ }^{23}$ As professional rugby players often endure planned collision-protected training, the incidence of such shoulder injuries leading to absence from play in rugby players is 90 times more likely to occur during competitive matches compared with club training sessions. ${ }^{3}$ Due to the high tackle rates in their game play, back-row players have been reported to have the greatest incidence of shoulder injury of all rugby players, with one event reported per 37 hours of play. ${ }^{24}$ In addition, rugby players with glenohumeral dislocations proceed to experience further dislocations and subsequent instability in nearly two thirds of cases and often require surgical stabilization in up to $50 \%$ of cases, ${ }^{3}$ with professional rugby players subsequently continue to pursue playing rugby poststabilization. $^{25}$ Similarly, our study demonstrated significantly greater frequencies of glenohumeral bone loss, degenerative changes, bicipital tendon lesions, and acromioclavicular joint degeneration on MRA in professional rugby players undergoing surgical stabilization for glenohumeral instability when compared with their nonprofessional counterparts. Such findings are concerning for rugby players and other collision athletes whom experience major glenohumeral trauma and subsequent instability at such young ages. Therefore, significant player counseling in relation to the extensive pathologies acquired in collision sports is required in the player hoping to return to play following surgical stabilization.

\section{Limitations}

This study had several limitations. First of all, this study has all the disadvantages inherent to retrospective evaluations. Second, the radiologist was aware that each patient had a clinical diagnosis of glenohumeral instability, which may lead to reporting bias. Furthermore, no control group was used in this study.

\section{Conclusions}

Rugby players undergoing surgical stabilization for glenohumeral instability often have a significant number of pathologies identified on MRA at the time of surgery. Professional rugby players tend to have concerningly greater frequencies of findings when compared with nonprofessional rugby players.

\section{References}

1. Galvin JW, Ernat JJ, Waterman BR, Stadecker MJ, Parada SA. The epidemiology and natural history of anterior shoulder instability. Curr Rev Musculoskelet Med 2017;10:411-424.

2. Gibbs DB, Lynch TS, Nuber ED, Nuber GW. Common shoulder injuries in American football athletes. Curr Sports Med Rep 2015;14:413-419.

3. Headey J, Brooks JH, Kemp SP. The epidemiology of shoulder injuries in English professional rugby union. Am J Sports Med 2007;35:1537-1543.

4. Kawasaki T, Ota C, Urayama S, et al. Incidence of and risk factors for traumatic anterior shoulder dislocation: An epidemiologic study in high-school rugby players. J Shoulder Elbow Surg 2014;23:1624-1630.

5. Owens BD, Agel J, Mountcastle SB, Cameron KL, Nelson BJ. Incidence of glenohumeral instability in collegiate athletics. Am J Sports Med 2009;37:1750-1754.

6. Owens BD, Duffey ML, Nelson BJ, DeBerardino TM, Taylor DC, Mountcastle SB. The incidence and characteristics of shoulder instability at the United States Military Academy. Am J Sports Med 2007;35:1168-1173.

7. Sconfienza LM, Albano D, Messina C, Silvestri E, Tagliafico AS. How, when, why in magnetic resonance arthrography: An International Survey by the European Society of Musculoskeletal Radiology (ESSR). Eur Radiol 2018;28:2356-2368.

8. Murphy CP, Frangiamore SJ, Mannava S, et al. Effect of anterior glenoid labral tears and glenoid bone loss at the NFL combine on future NFL performance. Orthop J Sports Med 2018;6:2325967118784884.

9. Chandnani VP, Yeager TD, DeBerardino T, et al. Glenoid labral tears: Prospective evaluation with MRI imaging, MR arthrography, and CT arthrography. AJR Am J Roentgenol 1993;161:1229-1235.

10. Gissane C, Jennings D, Kerr K, White J. Injury rates in rugby league football: Impact of change in playing season. Am J Sports Med 2003;31:954-958. 
11. Haseler CM, Carmont MR, England M. The epidemiology of injuries in English youth community rugby union. $\mathrm{Br} J$ Sports Med 2010;44:1093-1099.

12. King DA, Hume PA, Milburn PD, Guttenbeil D. Match and training injuries in rugby league: A review of published studies. Sports Med 2010;40:163-178.

13. Hill NE, Rilstone S, Stacey MJ, et al. Changes in northern hemisphere male international rugby union players' body mass and height between 1955 and 2015. BMJ Open Sport Exerc Med 2018;4:e000459.

14. Garraway WM, Lee AJ, Hutton SJ, Russell EB, Macleod DA. Impact of professionalism on injuries in rugby union. Br J Sports Med 2000;34:348-351.

15. Gianotti S, Hume PA, Hopkins WG, Harawira J, Truman R. Interim evaluation of the effect of a new scrum law on neck and back injuries in rugby union. Br J Sports Med 2008;42:427-430.

16. Crichton J, Jones DR, Funk L. Mechanisms of traumatic shoulder injury in elite rugby players. Br J Sports Med 2012;46:538-542.

17. Horsley IG, Fowler EM, Rolf CG. Shoulder injuries in professional rugby: A retrospective analysis. J Orthop Surg Res 2013;8:9.

18. Montgomery C, O’Briain DE, Hurley ET, Pauzenberger L, Mullett H, Moran CJ. Video analysis of shoulder dislocations in rugby: Insights into the dislocating mechanisms. Am J Sports Med 2019;47:3469-3475.
19. Mannava S, Frangiamore SJ, Murphy CP, et al. Prevalence of shoulder labral injury in collegiate football players at the National Football League scouting combine. Orthop J Sports Med 2018;6:2325967118783982.

20. Cohen SB, Towers JD, Bradley JP. Rotator cuff contusions of the shoulder in professional football players: Epidemiology and magnetic resonance imaging findings. Am J Sports Med 2007;35:442-447.

21. Murphy CP, Frangiamore SJ, Mannava S, et al. Effect of posterior glenoid labral tears at the NFL combine on future NFL performance. Orthop J Sports Med 2018;6: 2325967118787464.

22. Knapik DM, Gebhart JJ, Sheehan J, Tanenbaum JE, Salata MJ, Voos JE. Recurrent labral tearing on magnetic resonance imaging is not predictive of diminished participation among National Football League athletes. Arthroscopy 2018;34:66-72.

23. Brooks JH, Fuller CW, Kemp SP, Reddin DB. Epidemiology of injuries in English professional rugby union: Part 1 match injuries. Br J Sports Med 2005;39:757-766.

24. Sundaram A, Bokor DJ, Davidson AS. Rugby Union onfield position and its relationship to shoulder injury leading to anterior reconstruction for instability. J Sci Med Sport 2011;14:111-114.

25. Murphy AI, Hurley ET, Hurley DJ, Pauzenberger L, Mullett H. Long-term outcomes of the arthroscopic Bankart repair: A systematic review of studies at 10-year follow-up. J Shoulder Elbow Surg 2019;28:2084-2089. 